\title{
Multiscale temporal probing of elemental ultrafast magnetization dynamics in permalloy using High order Harmonics
}

\author{
M. Barthelemy, A. Maghraoui, G.Versini, M. Vomir, J.-Y. Bigot \\ CNRS - Université de Strasbourg, IPCMS UMR7504, 23 rue du Loess BP 43, 67034, Strasbourg, France
}

\begin{abstract}
Chemically selective magnetization dynamics is probed in $\mathrm{Ni} 80 \mathrm{Fe} 20$ with High order Harmonics over a large temporal scale. It is shown that the ratio between effective exchange interaction constants of each element can be retrieved experimentally.
\end{abstract}

\section{Introduction}

Over the past years, magnetization dynamics has been investigated by probing transitions metals at resonance such as $2 \mathrm{p}_{1 / 2} 2 \mathrm{p}_{3 / 2}$ L-edges using X-ray Magnetic Circular Dichroism at femtoslicing synchrotron facilities [1-3] or $3 p_{1 / 2} 3 p_{3 / 2}$ M-edges using the table top Transverse Magneto Optical Kerr Effect with High order Harmonics probe [4,5]. In particular, by probing core levels to $3 \mathrm{~d}$ bands, those methods give access to a chemical selectivity in alloyed transition metals and consequently are sensitive to the sub-lattices interaction processes during the transient magnetization dynamics. For example, it has been found that the inter-sub-lattice exchange interaction has to be taken into account if the demagnetization times of each sub-lattice are considered [5-7].

In this work, both the demagnetization and magnetization precession, induced by a $1.55 \mathrm{eV}$ pump in $\mathrm{Ni}_{80} \mathrm{Fe}_{20}$, are probed with High order Harmonics over a large time scale. This study reveals the importance of the inter-sub-lattice exchange interaction on the demagnetization times and precession relaxation rate. Moreover, one can deduct from those measurements the ratio between effective exchange interaction constants of $\mathrm{Ni}$ and Fe: $\mathrm{J}_{\text {eff }}{ }^{\mathrm{Fe}} / \mathrm{J}_{\text {eff }} \mathrm{Ni} \sim 4$.

\section{Experimental configuration}

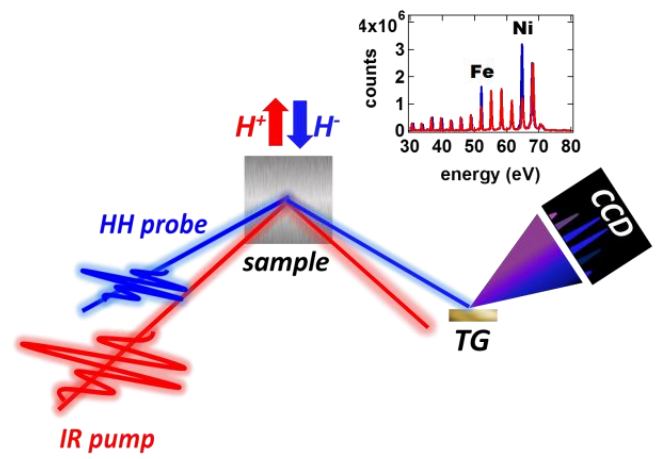

Fig. 1. Sketch of IR (1.55eV) pump and HHG probe (30-72eV) T-MOKE experiment. A $45^{\circ}$ incidence angle is set for the $\mathrm{HH}$ beam and a static magnetic field $\mathrm{H} \pm$ is applied in the plane of the sample. TG: toroidal grating. CCD: charge coupled device. Inset: Static reflected $\mathrm{HH}$ spectra measured on a $10 \mathrm{~nm} \mathrm{Ni} 80 \mathrm{Fe}_{20} / \mathrm{Al}_{2} \mathrm{O}_{3}$ thin film for antiparallel orientations of $\mathrm{H}$ showing the maximal magnetic contrast at the two Fe and Ni M-edges.

The sample is a $10 \mathrm{~nm}$ thick $\mathrm{Ni}_{80} \mathrm{Fe}_{20}$ film deposited on an $\mathrm{Al}_{2} \mathrm{O}_{3}$ substrate by ion beam sputtering. $\mathrm{A}$ static magnetic field $\mathrm{H}= \pm 2 \mathrm{kOe}$ is applied on the sample. On one hand, the demagnetization is obtained from measurements of the reflectivity with antiparallel transverse magnetic field (fig 1). On the other hand, the precession of the magnetization is measured for an angle of $10^{\circ}$ perpendicularly to the incidence and to the sample plane. The magnetization dynamics of both elements have been extracted from this TMOKE configuration by integrating spectrally over the harmonics H35 and H47 (corresponding respectively to Fe and Ni M-edges).

Corresponding author:bigot@unistra.fr and barthelemy@unistra.fr 


\section{Magnetization dynamics from demagnetization to magnetization precession}

Fig. 2a corresponds to the demagnetization of $\mathrm{Ni}$ and $\mathrm{Fe}$ in $\mathrm{Ni}_{80} \mathrm{Fe}_{20}$ for a magnetic field applied in the plane of the sample and respectively probed with harmonics $\mathrm{H} 45(66 \mathrm{eV})$ and $\mathrm{H} 35(54 \mathrm{eV})$ and pumped with a $4.7 \mathrm{~mJ} / \mathrm{cm}^{2}$ pump fluence. Fig. $2 \mathrm{~b}$ shows the magnetization precession of each element obtained when the magnetic field is rotated in the plane perpendicular to the incidence and to the sample plane by an angle of $10^{\circ}$.

a)

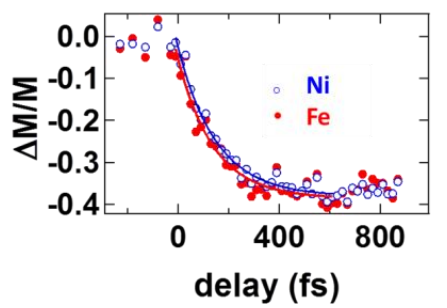

b)

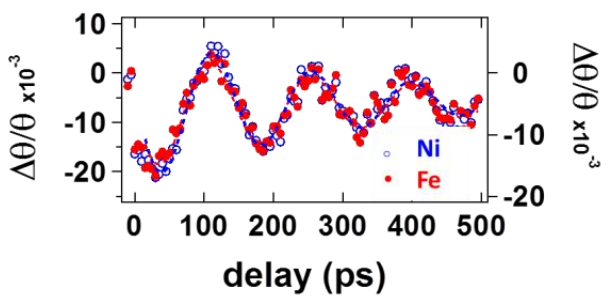

Fig. 2. T-MOKE signals probed in $\mathrm{Ni}_{80} \mathrm{Fe}_{20}$. a) Selective demagnetization of $\mathrm{Ni}(66 \mathrm{eV})$ and $\mathrm{Fe}(54 \mathrm{eV})$. b) Magnetization precession of Fe and Ni magnetizations.

The Landau-Lifshitz-Bloch equation associated to Langevin formalism can be used to deduce the demagnetization time $\tau_{M}{ }^{\varepsilon}$ attributed to each element $\varepsilon$ [7]. In particular, when $T<0.5 \mathrm{Tc}$, i.e. at low to intermediate temperature $\mathrm{T}$ compared to Curie temperature (Tc) of the considered ferromagnet, the ratio between demagnetization times can be expressed as follows:

$$
\frac{\tau_{M^{N i}}}{\tau_{M^{F e}}} \sim \frac{\mu_{N i} \alpha_{F e} \gamma_{F e} m_{0}^{F e} J_{e f f}^{F e}}{\mu_{F e} \alpha_{N i} \gamma_{N i} m_{0}^{N i} J_{e f f}{ }^{N i}}
$$

$\mu^{\varepsilon}(\varepsilon=\mathrm{Fe}, \mathrm{Ni})$ beeing the atomic magnetic momenta, $\alpha^{\varepsilon}$ are the Gilbert dampings of precession, $\gamma^{\varepsilon}$ the gyromagnetic ratios and $m_{0}^{\varepsilon}$ are the magnetizations at equilibrium, with $m_{0}^{\varepsilon}=\mu^{\varepsilon} \mathrm{N}^{\varepsilon}$ and $\mathrm{N}^{\varepsilon}$ is the number of atoms.

From our measurements, both elements demagnetize simultaneously with a characteristic time $\tau_{\mathrm{M}}{ }^{\mathrm{Ni}}=\tau_{\mathrm{M}}{ }^{\mathrm{Fe}}=142$ $\mathrm{fs}$, and the precession of both element is in phase, with same period $\left(\mathrm{T}_{\text {prec }}=150 \mathrm{ps}\right)$ and same Gilbert damping $\alpha^{\mathrm{Ni}}=\alpha^{\mathrm{Fe}}=350$ ps. The atomic magnetic momenta ratio is known to be $\mu^{\mathrm{Fe}} / \mu^{\mathrm{Ni}} \sim 4$. Finally the effective exchange interaction ratio can be deduced: $\mathrm{J}_{\text {eff }} \mathrm{Fe} / \mathrm{J}_{\text {eff }} \mathrm{Ni} \sim 4$. Moreover, the dependence of relaxation rate upon the pump density of excitation will be discussed considering a multiscale approach including temperature dependent exchange parameters.

\section{References}

[1] C. Stamm, T. Kachel, N. Pontius, R. Mitzner, T. Quast, K. Holldack, S. Khan, C. Lupulescu, E. F. Aziz, M. Wietstruk, H. A. Dürr and W. Eberhardt, Nature Materials 6, 740 (2007).

[2] C. Boeglin, E. Beaurepaire, V. Halté, V. López-Flores, C. Stamm, N. Pontius, H. A. Dürr and J.-Y. Bigot, Nature 465, 458 (2010).

[3] I. Radu, C. Stamm, A. Eschenlohr, F. Radu, R. Abrudan, K. Vahaplar, T. Kachel, N. Pontius, R. Mitzner, K. Holldack, et al., SPIN 5, 1550004 (2015).

[4] C. La-O-Vorakiat, M. Siemens, M. M. Murnane, H. C.Kapteyn, S. Mathias, M. Aeschlimann, P. Grychtol, R. Adam, C. M. Schneider, J. M. Shaw, et al., Phys.Rev. Lett. 103, 257402 (2009).

[5] S. Mathias, C. La-O-Vorakiat, P. Grychtol, P. Granitzka,E. Turgut, J. Shaw, R. Adam, H. Nembach, M. Siemens, S. Eich, et al., Proc. Natl. Acad. Sci. 109, 4792 (2012).

[6] A. J. Schellekens and B. Koopmans, Phys. Rev. B 87,020407 (2013).

[7] D. Hinzke, U. Atxitia, K. Carva, P. Nieves,O. Chubykalo-Fesenko, P. M. Oppeneer, and U. Nowak, Phys. Rev. B 92, 054412 (2015). 